RESEARCH ETHICS

\title{
The battering of informed consent
}

M Kottow

J Med Ethics 2004;30:565-569. doi: 10.1136/ime.2003.002949

Correspondence to: Professor M Kottow,

Escuela de Salud Pública

Facultad de Medicina

University de Chile,

Independencia 939,

Santiago, Chile;

kottow@hotmail.com

Received 30 January 2003

In revised form

12 March 2003

Accepted for publication

16 June 2003

\begin{abstract}
Autonomy has been hailed as the foremost principle of bioethics, and yet patients' decisions and research subjects' voluntary participation are being subjected to frequent restrictions. It has been argued that patient care is best served by a limited form of paternalism because the doctor is better qualified to take critical decisions than the patient, who is distracted by illness. The revival of paternalism is unwarranted on two grounds: firstly, because prejudging that the sick are not fully autonomous is a biased and unsubstantial view; secondly, because the technical knowledge of healthcare professionals does not include the ethical qualifications and prerogative to decide for others.

Clinical research settings are even more prone to erode subjects' autonomy than clinical settings because of the tendency and temptation to resort to such practices as shading the truth when consent to participation is sought, or waiving consent altogether when research is done in emergency settings.

Instead of supporting such dubious practices with unconvincing arguments, it would seem to be the task of bioethics to insist on reinforcing autonomy.
\end{abstract}

A basic tenet of medical ethics is that people whose body is to be interfered with, be it for therapeutic and/or research purposes, ought to be fully informed about the intended procedure prior to expressing their uncoerced, explicit, and revocable acceptance to participate. This interactive process was first proclaimed for research protocols in 1933, only to be shamelessly disregarded and violated by the German Nazi dictatorship. ${ }^{1}$ International recognition of the fundamental right to informed consent was initially obtained through the Declaration of Nuremberg (1947) and ratified by the successive Declarations of Helsinki (1964-2000) and others, extending it to patients' involvement in the institution, omission or suspension of medical treatment that directly concerns them.

Informed consent continues to be a desirable goal, but pragmatic reasoning stresses the circumstantial difficulties of fully respecting autonomy in clinical situations. ${ }^{2}$ The fully informed patient has been considered an exceedingly distant goal, attempts to reach it being bound to fail because sick people are emotionally unstable and their rational judgment impaired. The doctrine of therapeutic privilege is at times invoked to avoid disclosures under the pretext that they might be harmful to the patient. Disclosure to research subjects has been firmly defended in theory, although practical adherence has been lackadaisical at best. Investigations on human subjects have increasingly established a strategy of carrying out in underdeveloped host countries investigations that are sponsored and planned in developed nations. This shift has gone hand in hand with efforts to reduce information to research subjects, who are considered to be unable to understand disclosure to its full extent. Based on these considerations, a number of papers in recent years have made misguided efforts to erode the conceptual framework of such strong subject protection as is to be expected from complete and unbiased information. Part of the protection that had been gained in the name of autonomy was unobtrusively taken over by paternalistic medical attitudes which have tried to theoretically buttress the widespread practice of diminishing patients' participation in decisions that concern them. Unfortunately, First World bioethicists have been actively gnawing at the autonomy of the less fortunate social groups, those whom Sen characterises as deprived and limited in their capabilities. ${ }^{3}$
Autonomy is also eroded when subjects receive placebos or insufficient treatment in therapeutic clinical trials or are denied the benefits accruing from the study. These constitute so called "pragmatic" research ethics, whereby poor disclosure standards and other unacceptable conditions are offered to destitute populations on the presumption that poverty and lack of education entail the inability to exercise autonomy and make decisions.

\section{INFORMED CONSENT, INFORMED DECISION}

Informed consent in research may obey moral standards: sense $_{1}$, or legal requirements: sense ${ }_{2}$; these do not often coincide and pre-eminence is successfully claimed by the legal standard. Standards of disclosure have also been identified, classified, and criticised as: (a) professional practice standard-technical information; (b) reasonable person standard-what the subject would normally need to know in order to render an informed consent, and (c) actual/ subjective personal standard-the subject's avidness for information, which could become limitless. ${ }^{4}$ Reasonable person and subjective standards tend to conflate if the suggestion is accepted that each person may determine the level of information she desires and exceed the limit of what the informer considers reasonable. In the infant years of bioethics, it was widely believed that the patient was "entitled to information which is sufficient to provide him with the opportunity to make a rational decision", (Sen, ${ }^{3}$ p 197) as opposed, and to be preferred to the autonomy of unfettered requests for any potentially useful disclosure.

Informed participation of research subjects and patients was originally a value very dear to both clinical and research ethics. It is an ideal to be approached, ${ }^{5}$ its neglect is unacceptable. " $[\mathrm{F}]$ reedom and informed consent is central" to the procedure leading to a physician/patient contract, ${ }^{7}$ to the point that "the courts, in adjudicating disputes about the adequacy of informed consent, elevate the liberal principle of self determination to the highest and most decisive principle $^{\prime \prime} .{ }^{8}$ Investigators were reminded that " $[\mathrm{I}]$ informed consent has become the 'gold standard' for ethically responsible research with human subjects".

Abbreviations: CIOMS, Council for Organisations of Medical Sciences; IRB, institutional review board. 
Relying on the same concept of informed consent for both research and clinical situations is distorting and probably one of the major causes of the fraying of the edges of the principle of autonomy, especially if the matter is vaguely defined. Subjects entering a research protocol only exercise their free will when initially considering their participation and, again, if they eventually decide to opt out prematurely. But, as recruited research subjects they are no longer autonomous and must adhere to the requirements of the protocol without in any way being able to influence the way in which they participate. The situation differs when patients are presented with alternative courses of treatment in a clinical setting where they are able autonomously to choose the one most amenable to their desires, interests or way of life. $^{10}$ Furthermore, they remain free to change their mind during the course of treatment. Patients should not be expected to necessarily consent or agree to what the physician believes is best, nor to abide by the most commended standard of medical practice. Veatch has made the criticism that informed consent is not only a misnomer but a misleading proposition that extinguishes patient autonomy and the ethically "robust notion of active patient participation in the choice of any plausible alternatives". ${ }^{11}$ To avoid this pitfall, which certainly occurs frequently in clinical practice, it seems appropriate to speak of informed decisions in clinical situations and refer to informed consent in research ethics, a distinguishing nomenclature that more accurately describes the different role autonomy plays in research as compared to clinical settings. Acknowledgement can thus be made of the distinction between the clinical situation, where honouring the process of information should restrain physicians from pre-empting medical decisions and unduly obscuring the gamut of options available to the patient, and the research setting, where the purpose of full disclosure is to reaffirm subjects' acceptance or justify their rejection to participate in the investigation proposed.

Over the years the principle of informed consent in research on human beings has become an essential and vastly agreed upon element of research ethics, whereas it has often been neglected in clinical practice. The schism between research ethics standards in different settings widened as unethical practices in biomedical investigations were revealed and severely reprimanded in affluent countries, but often tolerated, even encouraged, in the Third World. In clinical practice, prevalent paternalism, although overtly rejected, may at times pay little more than lip service to patient autonomy, and restricted disclosure continues to be defended in the name of firm and efficient medical guidance. After 30 years of bioethics, autonomy and free decision making have proved to be precarious values, unevenly and unpredictably honoured both in research and clinical biomedicine, all the more so in developing countries, as has been explicitly revealed and denounced in Asian and Latin American countries. ${ }^{12} 13$

\section{THE DEBATE AROUND FULL VERSUS CONDITIONED DISCLOSURE}

In recent years a number of authors have argued against a too rigid doctrine of informed consent, advocating instead some bland form of "modern" or "limited" paternalism which would somewhat reduce patient autonomy or, according to others, reinforce it in a rather roundabout way. Unfortunately, suggestions of this nature have often been ad hoc, supporting clinical decisions in accordance with the doctor's view of what is best for the specific medical situation at hand, at the cost of degrading the unflagging protection patients ought to gain from informed participation. ${ }^{14}{ }^{15}$ There has been an awareness that fully informed consent could not be completely honoured in certain circumstances, such as emergencies, unconsciousness, emotional instability, or borderline mental competence, but these circumstances are exceptional and should be duly qualified. Where such circumstances do not apply the physician is not entitled to interfere with the patient's autonomous decision. Granting a sort of limited informed consent also made clear, unfortunately, that authorised limitations of disclosure under these circumstances create new forms of discrimination, for they are most frequently applied to patients belonging to ethnic minorities who are the most likely candidates to require emergency room procedures. ${ }^{16}$ In addition, such dispensations allow physicians to design clinical research protocols which disregard consent and rely on the approval of institutional review boards (IRBs) which are insufficiently equipped to grant such approval. ${ }^{17}$

The surgeon general of the US issued a ruling that exhorted physicians to honour consent in clinical practice, whereas investigators were compelled to obtain informed consent, ${ }^{18}$ and yet the full exercise of autonomy, especially in the area of research, has tended to erode. Although favouring full disclosure to research subjects, Beauchamp et al state: "Problems of information processing and questionable voluntariness are so nuanced in the case of some subjects that a valid consent is somewhere between unobtainable and highly questionable". ${ }^{19}$ Cultural gaps between sponsor country investigators and host country subjects are presented as insurmountable, and are further exaggerated by stressing the poor educational level of people devoid of any formal schooling. The gap certainly exists, but it is inadmissible to pretend to be closing it by resorting to incomplete and inadequate information..$^{21}$

As the inefficiencies in the doctor/patient interaction are used to demean informed consent and informed decision making, the frailness of the power of patients and subjects increases, producing incommensurably more harm than that caused by the inconveniences of a detailed informing process.

\section{THERAPEUTIC RESEARCH}

An intrinsic and unavoidable constraint on informed consent occurs in therapeutic research on patients who will be randomised between a promising new drug and currently existing/available unsatisfactory medication. If fully informed, subjects will become aware that they must cope with the dilemma of possibly being denied access to a hopefully efficient, perhaps life saving drug, should they find themselves, purely by chance, in the control group. ${ }^{22}$ Doubts become all the more perplexing if the control group receive a non-active placebo. They remain equally unprotected if they refuse to participate, thereby losing any access to the promising therapy under study. The least committed strategy has been to withhold some information in order to obtain consent, under the justification that randomised, double blind studies are the gold standard if one is committed to the rigours of science and unwilling to be ethically bound to obtain fully informed consent from subjects. Such intended lack of information has also, however, been used in single blind placebos employed in run in and washout periods of medication studies. ${ }^{23}$ Researchers are often convinced that they will cull significant data and reach biomedical truths more readily if they practise some sort of deceit or somehow distort the informed consent process. Bok has called attention to this "shading of the truth", rejecting such practices with two powerful arguments: firstly that the institution of trust is morally undermined and loses effectiveness in such a sensitive area as the biomedical practices; and, secondly, agents damage their moral self esteem and risk presenting an increasingly soiled image of their own moral stance as they continue to practise deception. ${ }^{24}$ 
Commenting on the 1993 ethical guidelines of the Council for International Organisations of Medical Sciences (CIOMS), Levine writes: "These requirements are very much more protective against exploitation of inhabitants of developing countries than the more familiar ethical justifications such as informed consent that get so much more attention". ${ }^{25}$ But the CIOMS norms under scrutiny are vague and have not been consistently honoured, leaving research subjects involved in therapeutic investigations all the more unprotected if informed consent receives a cold shoulder. Critics have suggested that the CIOMS guidelines need to be "revised for internal consistency", ${ }^{26}$ for some of them are "not strong enough to prevent exploitation". ${ }^{27}$ Consequently, some research ventures-for example, the use of reduced zidovudine (AZT) therapy during pregnancy-were carried out in "direct violation of the Declaration of Helsinki of the World Health Organization and of the principle of informed consent". ${ }^{28}$ In fact, when CIOMS says that "[E]very effort will be made to secure the ethical imperative that the consent of individuals be informed ...", ${ }^{29}$ it is blandly accepting that researchers may just go deep enough into the hinterland to be spared anything more than perfunctory efforts at disclosure.

New guidelines issued by CIOMS in 2002 may perhaps be going too far in the opposite direction. ${ }^{30}$ Under the heading of "essential information" that "the investigator must provide", the document lists 27 items which, if fully honoured, will probably lead to a messy and confusing procedure, thus failing to achieve the purpose of adequate disclosure and tempting researchers to work in poor areas with destitute subjects and to use ethically impermissible methods. Requiring exposure of "the expected benefits of the research to the community or the society at large, or contributions to scientific knowledge" (guideline 5, no 11), for example, seems a disproportionately large mandate for every prospective subject. Nor can all researchers be expected to be candid about research that will not benefit the host country. Each item of the CIOMS guidelines is important, but not all of them are relevant in every research situation.

The UNAIDS guidance document (2000) lists, among other conditions that may lead to exploitation of research subjects in the Third World, the limited ability of individuals to understand the informed consent process, and the limited ability of individuals to be able to freely give their informed consent in the light of prevailing class, gender, and other social and legal factors. ${ }^{31}$ What UNAIDS does not mention is researchers' limitations and their unfulfilled duty to adapt their own use of language to the comprehension level of the subjects with whom they wish to communicate.

In spite of Tuskegee Valley, Willowbrook, and other fiascos in ethical research, respected actors in the field, such as Beecher and Koski, believe that "the only true protection afforded research subjects comes from a well trained, well meaning investigator". ${ }^{32}$ Since vulnerable subjects cannot expect all investigators to be sufficiently well trained, and since it is not easy to agree upon what values a well meaning agent might respect, bioethics should instead insist that guidelines be developed which prohibit the deception of patients' and subjects' and any restriction of their autonomy, and which promote standard criteria for the administration of all trials.

\section{THE CURRENT EROSION OF AUTONOMY}

Major changes in the way research projects are conducted in recent years have led to the severe curtailment of subjects' protection. Biomedical investigation has massively shifted away from academic institutions to profit oriented corporations. Budgets have tightened and much work in the field is now located in Third World countries, some of them so poor that they lack medical facilities. This had led sponsor institutions to feel free to deny more than minimal compensation, and also to deny the granting of any postresearch benefits, arguing that the host community had no healthcare to begin with. Cultural differences and local educational deficiencies have served as excuses to relax ethical standards to the point of introducing such nomenclature as "aspirational ethics"-required in developed nations, and "pragmatic ethics" which is an anaemic version of "good enough for the Third World". ${ }^{33}$ Merely acknowledging the existence of a double ethical standard already goes a long way to justifying it (Macklin, ${ }^{31}$ pp 17-36). The ensuing ethical laxness has backfired and finally infected research protocols in developed countries also, leading to the waiver of informed consent in research in emergency settings, as well as introducing other "serious shortcomings in the process of informed consent". ${ }^{34}$

Many of the criticisms aimed at informed consent have addressed either structural problems of the process itself, or contextual excuses for abridging it. More recently, a number of authors have gone so far as to debunk informed consent on the grounds that patient/subject autonomy can be better preserved by delegating decisions to institutions such as IRBs. $^{36}$ Clinical medicine has witnessed the defence of distributing quinacrine in the Third World—a sterilising drug prohibited in the US because it may induce cancer:

The duty to respect persons includes the duty to respect their autonomous decisions regarding the use of nonapproved medical products, even if they lack some information about the possible consequences of such use, or if the relationship between the participants exchanging products is capitalistic.

And:

[I]n the case of selling or giving away unapproved medical products, if the individuals autonomously choose to be engaged in the enterprise, even though they may not be as knowledgeable as some opponent would like them to be, then there is no moral justification to prevent the transaction from occurring (Cooley, ${ }^{39} \mathrm{p} 437$ ).

Disclosure to potential users is bluntly thwarted for no other reason than to salvage profits, hiding even highly sensitive information about severe potential risks. The highly explosive policy of uninformed autonomy is thus employed to the clear advantage of providers.

There are admittedly various stakeholders in the research process, and some authors are concerned that "strict 'autonomy protecting' rules associated with the moral standards of sense $_{1}$ disclosure may impose unfair burdens upon institutions, researchers, and health care professionals". ${ }^{35}$ Family ties, cultural idiosyncrasies and the overall pursuit of knowledge have also been invoked as stakeholders whose interests should not be neglected. Before lamenting unfair disadvantages for institutions with which they sympathise, scholars should be more concerned about the burdens loaded upon the research subjects who are immensely more fragile and susceptible to harm. ${ }^{36}$ Informed consent was devised to protect research subjects, just as informed decision is expected to highlight patients' right to autonomy. To protect doctors from litigation-happy patients, efforts were deployed to discredit fully enlightening disclosure, at the price of limiting patients' autonomy and protection. ${ }^{37}$ Beyond serving to disguise a paternalistic attitude, submissive consent serves to safeguard doctors against the pressures of malpractice, for the patient appears to have taken upon himself the possible risks of treatment being offered..$^{10}$ 
The idea that the interests of research sponsors and institutions are legitimate enough to allow a reduction of ethical rigour is unfortunately gaining new force. Pullman has recently acknowledged that providing too much information to research subjects may be deleterious to the interests of investigators, so a balance should be sought between knowledge made available to decision makers and the various interests involved. The sudden change in nomenclature from subjects to decision makers occurs because IRBs are being suggested as the appropriate institutions to "provide a proxy consent on behalf of research subjects" who are technically insufficiently versed to understand a full disclosure. "If informed consent to research is to remain the ethical sine qua non of research ethics, in many cases the IRB is the only body capable of providing it" (Pullman, ${ }^{35}$ p 538). Ethics committees have been called upon to intercede in favour of simplified consent forms that respect local values and idiosyncrasies at the cost of full disclosure. The work of IRBs has been very diversely evaluated in many different ways, but there seems to be some consensus that IRBs have not been strong enough in supporting the right of research subjects to informed consent, with the result that there has been a lack of fully informed participants. Curiously enough, in spite of the shortcomings of IRBs having been recognised ("IRBs arguably have experienced a dulling of their moral sensibilities" $)^{38}$ they continue to be defended as the most reliable monitor of ethics in research. Thus, individual autonomy of research subjects is emasculated.

\section{COMPROMISES}

Well meant efforts to safeguard and retain some authority for the physician, which at the same time stress her duty to both inform and help patients in their decisions, have suggested that information should not be restricted to facts but should also include value clarification and the presentation of the views held by the doctor. In the final analysis the decision should remain with the patient, but the doctor will have taken the opportunity to present facts and discuss values, in order to improve the quality of decisions reached by exercising a "rational non-interventional paternalism". ${ }^{39}$ Underlying this approach is the premise that the doctor is sufficiently well trained to assess and discuss patients' values, a presupposition that needs further analysis in view of the trend towards providing training for healthcare providers which, while highly sophisticated and technical, is as yet still shaky in terms of ethics. In the same vein, the suggestion has been made that patients holding non-rational beliefs should be guided toward better reasoned views in order to clarify and disinfect their beliefs, thus helping them make rational choices. ${ }^{40}$ Even such soft paternalism has not gone unchallenged by those arguing that patients' existential autonomy-defined as the act of making a decision-is at stake unless healthcare agents step back to the role of informers/advisers, in order to fully respect patients' autonomy. ${ }^{41}$ Others have argued that the concept of consent "necessitates a dominant/subordinate type of relationship, or the type of relationship that conflicts with the ideas of liberty and autonomy which consent is meant to create" (Habiba, ${ }^{10}$ p 185).

\section{CONCLUDING REMARKS}

The value of informed consent in research on human subjects and informed decision making in clinical settings is being eroded as a result of having come under constant fire, some of it seriously analytical but most of it heavily influenced by ad hoc considerations that seek to reduce patient/subject autonomy in order to expedite biomedical investigations and make clinical decisions easier. The importance of unencumbered participation has slowly gained recognition in recent decades but is now in danger of being swept away by criticism, vested interests, scientific hubris, and ethical neglect. The core value of informed consent and informed decision making must be constantly reviewed and defended, even at the risk of triteness. A strong defence could be built, I suggest, on the following five propositions.

1. Clinical patients are entitled to all the necessary information available to help them decide between possible courses of action or omission. The suspicion that a patient might reach a medically unsound decision, or one that apparently collides with his interests, is no excuse for withholding information or distorting the facts. The idea of informed decision making is incompatible with incomplete knowledge, for how is one to decide in ignorance of all valid options?

2. In therapeutic research, patients become subjects and should be made aware that they will be treated as such-that is to say, impersonally and in accordance with the sober subject/investigator relationship, which precludes the warm and compassionate interaction between the sick and their healthcare providers.

3. Third party interests that might collide with the needs and protection of research subjects are unacceptable excuses for withholding sensitive information. One suggested way of compensating for less than optimal disclosure has been to pay subjects for their participation, but this introduces the complex and highly controversial issue of vulnerability and exploitation. In addition to learning about risks and benefits, research subjects should be granted information privileges about the purpose of the project if they so desire; they are equally entitled to know who will benefit from their participation. There is no justification for withholding information that might have influence, now or in the future, on patients'/subjects' interests. Admittedly, there is on the other hand no need to give information beyond what is liable to affect them.

4. In vitro research with material provided by the human body-bodily fluids, biopsy material, amniotic membrane, placenta, genes-needs detailed clarification as to the data that are being sought: could they in any way become deleterious to the provider of the material? In a protocol reviewed some time ago, meconium was to be studied for traces of drugs possibly abused by the mother. This could have yielded unwanted revelations about the woman's habits and created a conflict of confidentiality. In other circumstances, data culled might be critical to, and unwanted by, the subject, as occurs, say, in Huntington's disease when unexpected detection of genetic burdens is unveiled.

5. In vivo research performed on a subject's body requires information about methodology and biology. Why placebos and not current best treatment? Under what circumstances will unblinding be necessary? Research subjects, especially if they are patients, ought to be made familiar with the essentials of equipoise, so they can understand why a given therapy is being compared to an unknown new agent. The essentials of double blind randomisation should also be explained so that the patient is aware of the chance of being placed in the placebo arm of the trial and the attendant risks, and also of the additional risks involved should she find herself in the arm of the trial in which she will receive the new, unproven medication instead of standard therapy. Biological information raises the well known questions of probability and the extent of ill effects and benefits, but information should also be provided about latent effects which might appear at a later date; the possible 
future use of culled data, and the chances of storing remnant samples and eventually using them in other, as yet unplanned, investigations-or the assurance should be given that this will not happen. If an individual is deemed unable to grasp the essential strategies of biological research that have a bearing on his/her participation, he/she should not be recruited.

Judged on the basis of these premises, the so called professional standard of information is obviously too sparse and should not be employed; a subjective standard, idiosyncratically chosen by the patient is, on the other hand, too ample and unfocused, whereas a reasonable standard will be exceedingly vulnerable to a personalised agenda. For lack of a better name, a pathic/proleptic standard is here suggested, whereby the patient or research subject receives all the information that may at any time be expected to affect himthe pathic element-during the course of the investigation or therapy in question, including possible anticipated future influences on his interests - the proleptic element. This proposal differs somewhat from the reasonable standard, where it is the informer who sets the standard of what is reasonable. With the pathic/proleptic standard it is the subject who decides how she may be affected by entering a research project, but it remains the duty of the informer to lay out the possible future consequences for her of the present investigation and to inform her also of what will be done with the material gathered.

These conclusions are bound to be resisted or simply dismissed. They do propose a highly regulated, and possibly unrealistic, exchange of information, and will surely be rejected by hard core scientists, paternalistic physicians, and interested third parties. Nevertheless subjects and patients must be protected, because it is perverse for biomedical practice to support regressive attitudes toward safeguarding the weak and defending the autonomy of individuals. It is also worrying to observe how bioethics is becoming ever more sympathetic to the interests of the strong and the mighty, offering arguments that favour sponsor countries, research institutions, biomedical big business, and career minded investigators. Patients and research subjects must continue to be the main concern of ethics, taking care to eliminate any and all weaknesses and deficiencies from the process of providing complete and pertinent information to ensure protection of the vulnerable and unfailing respect for their autonomy.

\section{REFERENCES}

1 Sass HM. Rundschreiben 1931: Pre-Nuremberg German regulations concerning new therapy and human experimentation. J Med Philos 1983;8:99-112.

2 Kirby MD. Informed consent: what does it mean? J Med Ethics 1983;9:69-75 3 Sen A. Inequality re-examined. Cambridge MA: Harvard University Press, 1995.

4 Strong C. Informed consent: theory and policy. J Med Ethics 1979;5:196-9.
5 Brody H. Ethical decisions in medicine. Boston: Little, Brown and Co, 1976:51. 6 Beauchamp TL, Childress JF. Principles of biomedical ethics [2nd ed]. New York/Oxford: Oxford University Press, 1983:66.

7 Engelhardt Jr HT. The foundations of bioethics. New York/Oxford: Oxford University Press, 1986:251.

8 Veatch RM. A theory of medical ethics. New York: Basic Books, 1981:45.

9 Wicclair MR. Informed consent and research involving the newly dead. Kennedy Inst Ethics J 2002;12:351-3, 372.

10 Veatch RM. Abandoning informed consent. Hastings Cent Rep 1995;25:5-12.

11 Quo RZ. GAT has bioethics to offer the developing countries. Bioethics 1993;7:108-25.

12 Luna F. Paternalism and the argument for illiteracy. Bioethics 1995;9:283-90.

13 Weiss GB. Paternalism modernised. J Med Ethics 1985;11:184-7.

14 Habiba MA. Examining consent within the patient-doctor relationship. J Med Ethics 2000;26:183-7.

15 Komrad MS. A defence of medical paternalism: maximising patient's autonomy. J Med Ethics 1983:9:38-44.

16 Silverman WA. The myth of informed consent in daily practice and in clinical trials. J Med Ethics 1989;15:6-11, at 7.

17 Dula A. Bearing the brunt of the new regulations: minority populations. Hastings Cent Rep 1997;27:11-12.

18 Katz J. Blurring the lines: research, therapy, and IRBs. Hastings Cent Rep 1997;27:9-11.

19 Beauchamp TL, Jennings B, Kinney ED, et al. Pharmaceutical research involving the homeless. J Med Philos 2002;27:547-64, 557-8.

20 Cox C, MacPherson CNL. Modified informed consent in viral seroprevalence study in the Caribbean. Bioethics 1996;10:222-32.

21 Resnik DB. The ethics of HIV research in developing nations. Bioethics 1998;12:286-306

22 Hewlett S. Consent to clinical trials: preliminary study of the views of prospective participants. J Med Ethics 1996;22:232-7.

23 Evans M. Justified deception? The single blind placebo in drug research. J Med Ethics 2000;26: 188-93.

24 Bok S. Shading the truth and seeking informed consent for research purposes. Kennedy Inst Ethics J 1995;5:1-17.

25 Levine RJ. The "best proven therapeutic method" standard in clinical trials in technologically developing countries. J Clin Ethics 1998;9:167-72, 170.

26 Levine C. Placebos and HIV. Lessons learned. Hastings Cent Rep 1998;28:43-8 at 45 .

27 Glantz LH, Annas GJ, Grodin MA, et al. Research in developing countries: taking "benefit" seriously. Hastings Cent Rep 1998;28:38-42 at 39.

28 Clark PA. The ethics of placebo controlled trials for perinatal transmission of HIV in developing countries. J Clin Ethics 1998;9:156-66 at161.

29 Grady C. Science in the service of healing. Hastings Cent Rep 1998;28:34-8 at 37.

30 Council for International Organisations of Medical Sciences. Ethical guidelines for biomedical research involving human subjects. Geneva: CIOMS, 2002:19-20.

31 Macklin R. After Helsinki: unresolved issues in international research Kennedy Inst Ethics J 2001;11:17-36 at 33.

32 Moreno JD. Goodbye to all that: the end of moderate protectionism in human subject research. Hastings Cent Rep 2001;31:9-17 at 16.

33 Cooley D. Good enough for the Third World. J Med Philos 2000;25:427-50.

34 Mastroiani A, Kahn J. Swinging on the pendulum: shifting views of justice in human subjects research. Hastings Cent Rep 2001;31:21-8.

35 Pullman D. Conflicting interests, social justice and proxy consent to research. J Med Philos 2002;27:523-45.

36 Kottow M. The vulnerable and the susceptible. Bioethics. In press.

37 Gillet G. Informed consent and moral integrity. J Med Ethics 1989;15:117-23.

38 Eckenwiler L. Moral reasoning and the review of research involving human subjects. Kennedy Inst Ethics J 2001;11:37-69.

39 Savulescu J. Rational non-interventional paternalism: why doctors ought to make judgments of what is best for their patients. J Med Ethics 1995;21:327-31

40 Savulescu J, Momeyer RW. Should informed consent be based on rational beliefs? J Med Ethics 1997;23:282-8.

41 Madder H. Existential autonomy: why patients should make their own choices. J Med Ethics 1997;23:221-5. 